\title{
Learning single alternation of running speeds in a runway without handling between trials'
}

JOYCE H. HARRIS AND GARTH J. THOMAS 2

UNIVERSITY OF ILLINOIS

Rats were able to learn alternation of fast-slow speed of running in response to alternation of reward-nonreward in a runway task ev'n though they were not handled between trials and food was not presented in the goalbox until after each run. These results were interpreted as evidence that the animals could make use of cues more subtle than either of these in order to learn the sequential responses necessary in a single alternation task.

There has been some question recently (Surridge \& Amsel, 1965a, b; Capaldi \& Spivey, 1965) about whether or not the alternating pattern of running speeds in a runway which rats learn in response to alternating reinforcement is attributable to the seqence of reinforced and nonreinforced trials and to nothing else. Surridge \& Amsel (1965b) presented data showing that rats can learn to run faster on reinforced trials even if no regular pattern of reinforcement is used. Their Ss apparently were able to discriminate reinforced trials from nonreinforced ones on the basis of unknown cues. The fact that rats can learn from such unintended cues, i.e., that the cues are sufficient for learning, does not mean that they are necessary for learning. It does, however, emphasize the fact that the experimental conditions must be very carefully controlled.

One source of cues which was not eliminated in any of the previous studies was possible inadvertant differential treatment by $\mathrm{E}$ in the course of handling Ss between trials, i.e., taking them out of the goal box, placing them in the start box, etc. In the present study Ss were not handled between trials. In addition, no visual or olfactory cues were supplied by the food itself, since food was not presented in the goal box until after the animal had made its run.

Method

Sixteen male albino rats (Holtzman) that averaged 247 gm in weight at the beginning of the experiment were used as Ss. The apparatus consisted of three straight and equal runways joined together to form a triangle with a compartment which was half goal box and half start box at the vertex of each angle. The alleys were 56 in. long, $3-1 / 4$ in. wide and $5-1 / 4$ in. high. The shape of the compartment at the vertexes resembled a 5-sided "home plate" with the point of the home plate having a $120^{\circ}$ angle which pointed toward the center of the triangle. The runway troughs butted into the sides of this angle to form the $60^{\circ}$ angle of the equilateral triangle. The back wall of the boxes was 13-1/2 in. long; the side walls were 13 in. long; and the walls forming the $120^{\circ}$ angle were 8 in. long. All walls were 5-1/4 in. high. These three boxes were each divided in half by a partition extending from the vertex of the $120^{\circ}$ angle to the center of the back wall. The left half was considered as the goal box and the right half the start box. A guillotine door separated these two halves. The Ss were run around the triangular runway in a clockwise direction. Three guillotine doors, one at the end of one runway, one separating the goal box from the start box, and one at the beginning of the next runway, could be operated from a waist-high pedestal at each corner of the triangle. A low partition (1-3/4 in. high) extended across the back of the goal boxes to form the eating area where the food pellets were delivered. A flexible Tygon tube connected a hole in the platform of the pedestal with the eating area of the goal box, and a hand-operated food dispenser was situated over the hole. The runways and start boxes were painted flat black and the goal boxes were flat white. The entire apparatus was covered with transparent Plexiglas. The runways and start-goal boxes rested on the floor. The $E$ stood in the center of the triangle formed by the runways and operated the doors and food dispensers from the pedestals. Running times to traverse a runway were measured with a stopwatch and were transformed to running speeds (reciprocals x 100).

Six days prior to the first day of training Ss were put on a 23-hr. food-deprivation schedule, and for two days before training they were given a number of the small reinforcement pellets (Noyes, 45-mg pellets) during their eating period in their home cages. The training period began when $S$ was taken from its home cage and placed in one of the start boxes. After $3 \mathrm{sec}$. the door at the start of the runway was opened. The door was closed after $S$ as soon as it entered the runway. The $E$ opened the door at the end of the runway for $S$ to enter the goal box, and, after $S$ entered the goal box, E simultaneously closed the door and turned the food dispenser. If the trial were reinforced $(R)$, the five Noyes pellets in the food-dispenser would fall down the tubing into the goal box eating area; if it were a nonreinforced (NR) trial, the food dispenser would be empty and no food would fall. In this way, E's actions were the same on both reinforced and nonreinforced trials. After $S$ had been in the goal box $20 \mathrm{sec}$. the door between the goal and start boxes was opened; after $S$ had been in the start box 3 sec. the door to the next runway was opened. Similar procedures occurred at each compartment at the points of the triangular runway. Each run down a runway was considered as one trial. The minimum interval between the time 


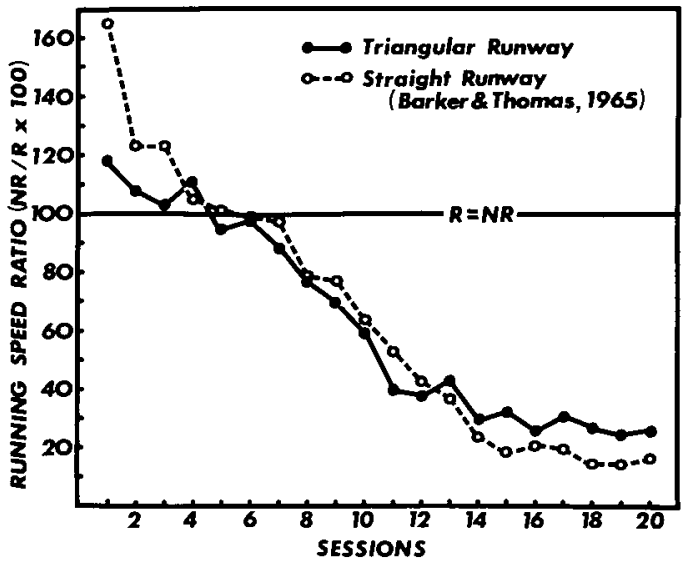

Fig. 1. Mean daily running speed ratios on both the triangular nuway and a straight runway. (The horizontal line at 100 on the ordinate indicates the point.at which speed on $N R$ trials equals speed on $\mathbf{R}$ trials. Above the line, $\mathbf{N R}$ is greater than $\mathbf{R}$, and below the line, $R$ is greater than $N R$.)

of reinforcement and the beginning of the next trial was $23 \mathrm{sec}$. The interval, however, was often longer because it depended upon how soon the animal moved to the next compartment after the door was opened. For the first four days of training Ss were given seven trials per day, and for the remaining 18 days they were given 13 trials per day. As the number of trials per day was not evenly divisible by 3 , the starting compartment each day precessed around the triangle. Every odd numbered trial (counting from the beginning of training) was reinforced. Rats were put into and taken out of the triangular runway through the start boxes. After the daily sessions Ss were allowed 1-hr. access to dry Purina rat chow; water was provided ad lib in home cages.

\section{Resulis}

The ratio of mean speed on nonreinforced trials to mean speed on reinforced trials $(N R / R \times 100)$ was computed for each $\mathrm{S}$ for each day (the smaller the ratio the more pronounced the alternation). Figure 1 shows the mean daily running speed ratios (NR/R x 100) on sessions 1 through 20 (days 1 through 4 were combined into sessions 1 and 2). The horizontal line at 100 on the ordinate indicates the point at which running speed on $R$ trials equals speed on NR trials. Fifty is the point at which the speed on $R$ trials is twice as fast as the speed on NR trials. The dashed curve in Fig. 1 was taken from another study from this laboratory (Barker \& Thomas, 1965) in which a single alley runway was used for alternation training. In that experiment the rats were handled between trials, and 10 trials per day with $25 \mathrm{sec}$. between reinforcement and the next trial were given. As can be seen from Fig. 1, the curves are quite similar. For evaluation of alternation performance at the end of training, mean running speeds on reinforced trials were compared with mean running speeds on nonreinforced trials for each $\mathrm{S}$ over the last five sessions. There was no overlap between the mean running speeds on reinforced and nonreinforced trials.

\section{Discussion}

Results of this experiment clearly show that, notwithstanding the precautions taken, rats were able to learn single alternation. This finding demonstrates that handling between trials does not provide indispensable cues for delayed single alternation in a runway. Rats almost surely use any available cues in learning such a task. Probably, they rely most heavily on relatively obvious cues like olfactory cues, stimuli provided by E's placing the food cup, food on E's hands between trials, etc. However, if these cues are eliminated, the Ss may rely on more subtle cues such as discrimination of the seemingly longer duration of time in the goal box prior to reinforced trials. Just how subtle the cues must be before rats are unable to discriminate them is open to further research.

On the other hand, the data suggest that the rat is capable of using "symbolic" processes (memory) to "bridge the gap" in time between trials, and it is the rats' "memory" of reinforcement or nonreinforcement on the previous trial that provides the "cue" for running fast or slow.

\section{References}

Barker, D. J., \& Thomas, G. J. Ablation of cingulate cortex in rats impairs alternation learning and retention. J. comp. physiol. Psychol., 1965, 60, 353-359.

Capaldi, E. J., \& Spivey, J. E. Comment. Psychon. Sci., 1965, $3,110$.

Surridge, C. T., \& Amsel, A. Performance under a single alternation schedule of reinforcement at 24-hour intertrial intervals. Psychon. Sci., 1965a, 3, 131-132.

Surridge, C. T., \& Amsel, A. A "patterning" effect that seems unrelated to after-effects from reward and nonreward. Psychon. Sci., $1965 \mathrm{~b}, 3,373-374$.

\section{Notes}

1. Supported by Grant MH-01370 from the National Institute of Mental Health. The authors are indebted to David J. Barker for comments and suggestions.

2. Now at the Center for Brain Research, University of Rochester, Rochester, New York. 